window periods and false positive results. And clinics will need the ability to perform routine confirmatory testing, such as Western blot analyses, among patients with positive results. These measures, along with detailed educational materials in package inserts, must be part of the home-marketing strategy.

Another controversial issue is cost. Currently, laboratories pay $\$ 12$ to $\$ 17$ for each OraQuick kit. The company expects to raise the price if over-the-counter status is approved, but it is not sure by how much. Public health officials argue that the test must be affordable to reach high-risk populations. A pilot study, sampling 240 HIV-positive patients, showed that most would pay no more than $\$ 15$ for a test. $^{4}$

Despite concern about affordability and the potential for the misuse of results, there is still strong support for home HIV testing. During the recent rash of false positive results, officials emphasized that the test remains an excellent screening tool, and many experts argue that the only way to halt the spread of the AIDS epidemic in the United States is to make HIV tests as simple as home pregnancy tests. If those who spoke at the FDA panel meeting in November are heard, the 20-year wait for a do-it-yourself HIV test may soon come to an end.
Drs. Wright and Katz are residents in internal medicine at Brigham and Women's Hospital, Boston, and editorial fellows at the Journal.

1. Branson BM. Home sample collection tests for HIV infection. JAMA 1998;280:1699701.

2. Marks G, Crepaz N, Senterfitt JW, Janssen RS. Meta-analysis of high-risk sexual behavior in persons aware and unaware they are infected with HIV in the United States: implications for HIV prevention programs. J Acquir Immune Defic Syndr 2005;39:446-53.

3. Branson B. Changes in HIV testing practices and counseling recommendations. Presented at the FDA Blood Products Advisory Committee Meeting, Gaithersburg, Md., November 3, 2005

4. Spielberg F. Over the counter HIV testing: a technology whose time has come. Presented at the FDA Blood Products Advisory Committee Meeting, Gaithersburg, Md., November 3, 2005

\title{
Unfinished Business - Expanding HIV Testing in Developing Countries
}

\author{
Kevin M. De Cock, M.D., D.T.M.H., Rebecca Bunnell, Sc.D., and Jonathan Mermin, M.D., M.P.H.
}

W hen the Group of Eight (G8) major industrial countries (France, the United States, Britain, Germany, Japan, Italy, Canada, and Russia) made a commitment in July 2005 to work toward universal access by 2010 to the prevention and treatment of human immunodeficiency virus (HIV) infection and AIDS, the move brought to light an HIVtesting emergency: knowledge of serologic status is required for the appropriate targeting of services and interventions. The World Health Organization and the Joint United Nations Program on HIV/ AIDS recently published revised guidelines for HIV testing, ${ }^{1}$ but field experience in Africa indicates that testing must be greatly expanded. It seems clear that to maximize benefit, a public health approach to HIV testing and treat- ment - including case finding and testing of partners - should become the norm.

Meeting the targets for antiretroviral treatment that had been set for the end of 2005 would have required testing as many as 180 million persons worldwide annually, ${ }^{2}$ far exceeding the current rates. ${ }^{3}$ Current guidelines recommend offering testing to persons with symptoms and signs that are potentially attributable to HIV infection or AIDS. ${ }^{1}$ However, since advanced immunodeficiency can be clinically silent, it would be more effective to offer testing to all patients attending health care facilities in locations with a high prevalence of HIV infection. Botswana, with a prevalence of more than 35 percent among people 15 to 49 years of age, has initiated such routine testing at clinics and hospitals nationwide. Kenya is increasing routine testing of pregnant women, hospitalized patients, and patients with tuberculosis; with an "opt-out" approach, less than 20 percent have chosen not to be tested. With routine testing of inpatients in selected hospitals in Uganda, 95 percent of inpatients agreed to be tested, and the prevalence of HIV was discovered to exceed 50 percent.

Current guidelines restrict the use of routine testing to settings in which antiretroviral therapy is available. ${ }^{1}$ We believe that the recommendations should support routine testing wherever basic HIV care and prevention are available. Such a change would improve efforts at prevention, allow infected persons to receive care such as cotrimoxazole prophylaxis, and 
normalize HIV testing. Prophylaxis against opportunistic infections is within the reach of even the poorest countries, and the identification of those who will need it benefits the entire public health system. In the United States, it is cost-effective to provide routine testing and counseling to all patients in hospitals with a prevalence of HIV of more than 1 percent, a practice recommended by the Centers for Disease Control and Prevention (CDC); in Africa, the prevalence among hospitalized patients is often greater than 50 percent.

Providing testing to family members of infected persons is critical; 50 percent of the spouses of infected African adults are also infected. ${ }^{3}$ The testing of partners is the first step toward providing care for those who are infected - and protecting those who are not. Knowledge of HIV status is associated with reductions in high-risk behavior; and providing preventive services, including condoms to HIV-discordant couples, decreases the risk of transmission by 80 percent. ${ }^{4}$ The children of infected women have an increased likelihood of infection, and pediatric HIV infection may indicate the presence of infection in other family members. The identification and treatment to HIV-infected mothers are important for their children, since their risk of death is at least doubled if their mother dies.

The prevalence of HIV infection among adolescents, especially young women, is high in much of Africa. Counseling and testing among young people is an important preventive strategy, although it may be ethically and legally challenging. Although premarital HIV testing is not cost-effective where prevalence is low, it is an

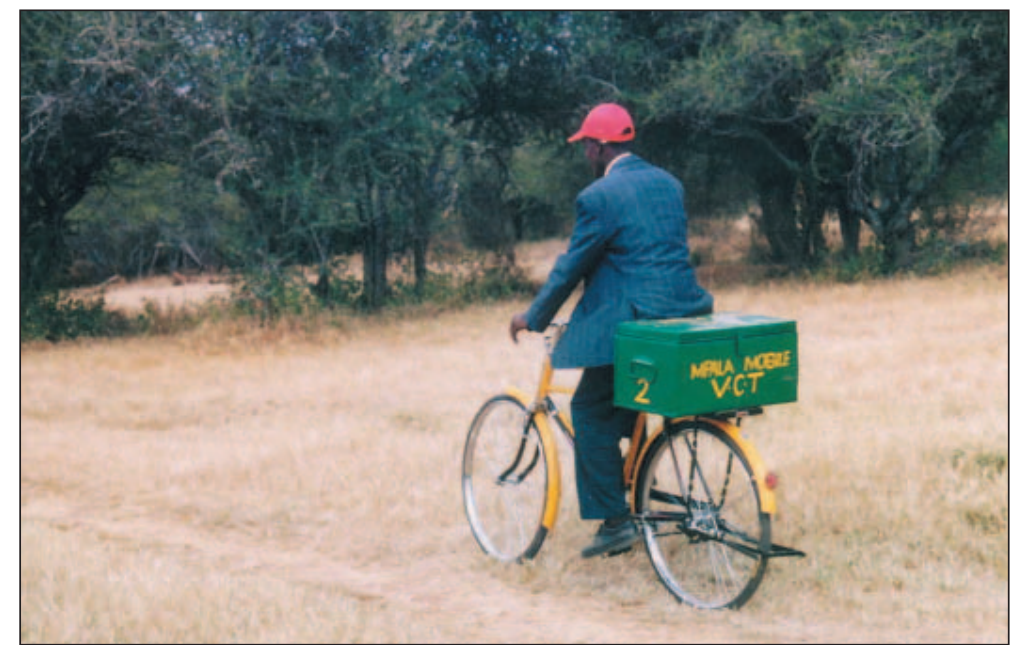

Traveling HIV Tester and Counselor in Kenya.

Service providers come by bicycle to remote areas with poor roads. In isolated desert regions, service delivery by camel is being developed.

important preventive practice in regions with generalized epidemics. Careful guidance is required, however, to determine how to implement such testing without coercion and how to limit the negative social consequences of a premarital diagnosis of HIV infection, especially for young women.

Approximately 2.3 million children worldwide are living with HIV infection, and children account for 18 percent of AIDSrelated deaths. Signs of HIV disease are often nonspecific, and more than half of infected African children die before two years of age. Antiretroviral therapy, therefore, must be started early, and universal HIV testing in pediatric clinical settings - an issue omitted from recent guidelines ${ }^{1}$ - will be required. Pediatric testing is complex: the possible presence of passively transferred maternal antibody limits diagnostic confidence until 18 months of age, and venipuncture is difficult. Moreover, exposure to HIV may continue during breast-feeding. Algorithms for testing children of various ages should be evaluated and endorsed by international public health authorities. For younger children, the algorithms could include rapid testing, with positive results verified by a central laboratory - a system being examined in Uganda and Kenya.

Since the denial of an HIV test may restrict an infected person's access to life-prolonging therapy, health care systems must determine whose role it is to grant permission for the testing of children, including orphans with no official guardians. In some countries, pediatric testing has been avoided for fear that HIV-infected children might be abandoned or neglected by families or caregivers, but this has not, to our knowledge, been documented. Guidance is also required with regard to the appropriate timing and methods of disclosing to children their HIV status.

In Africa, AIDS-related mortality is high among the providers of essential services - not only health care workers, but also teachers, police, and the military - so that stability, security, and economic development 
are all threatened. We recommend routine testing for HIV for persons in key occupations, with guarantees of confidentiality, protection against discrimination, free treatment for infected persons, and postexposure prophylaxis as appropriate.

The equitable expansion of HIV testing requires innovative approaches to reaching people in remote settings. New methods, such as finger-stick or salivary testing, are being used, and services are being provided in innovative ways, including testing of entire families in health care facilities and homes. Testing and counseling services have been delivered at community and religious institutions, youth centers, and military barracks. The shortage of health care professionals in Africa will have to be addressed in part by the training of community and lay workers to provide testing and counseling, treatment support, and other services.

Successful HIV-testing programs provide respectful and convenient service, confidentiality, accurate testing, full information, and effective referral. Rapid test algorithms involving the use of different tests in combination have a validity similar to that of standard approaches, ${ }^{5}$ do not require laboratory facilities or confirmation by Western blot assay, and can be performed by trained lay personnel. Performing rapid tests on fingerstick specimens in front of clients increases confidence in the results and prevents clerical errors. Tests may be performed sequen- tially or in parallel, with a third test used as a "tie breaker" for discrepant results. A subgroup of specimens may be sent to a laboratory for quality assurance.

Vigorous treatment and prevention programs limit the devastation wrought by AIDS, motivating a reassessment of attitudes toward HIV testing. The initial experience in Africa shows that the benefits outweigh the risks. According to Rose Apondi, a researcher at CDC-Uganda who studied HIV-infected persons in rural Uganda, home-based HIV testing and three months of antiretroviral therapy were associated with exclusively positive social experiences, such as community and partner support, in 79 percent of cases and with exclusively negative ones, such as stigma, in less than 1 percent.

HIV epidemics have also emerged in countries such as China, India, and Russia, where they are concentrated among injection-drug users, sex workers and their clients, and paid blood donors. Ensuring the appropriate distribution of services will require sentinel surveillance, followed by HIV testing targeted according to local epidemiologic trends. These countries have an opportunity that was not afforded to countries in Africa: before their epidemics become generalized, they may develop integrated strategies linking surveillance, prevention, HIV testing, antiretroviral therapy, and other care.

The increased resources available for combating HIV and AIDS, the strong emphasis on treatment, and the commitment of the G8 to universal access necessitate a massive expansion of HIV testing. The ideal that all citizens of high-prevalence countries should know their serologic status and should be tested repeatedly over the course of their lives should become explicit targets of preventive efforts. Increased rates of testing will, of course, generate other challenges, such as the provision of preventive services for HIV-infected persons, the disclosure of serologic status, and the notification of partners - all areas in which we must define the best practices for a new era.

\begin{abstract}
The opinions and statements in this Perspective are those of the authors and do not represent the official policy, endorsement, or views of the CDC, the U.S. Public Health Service, or the U.S. Department of Health and Human Services. Dr. De Cock is the director of CDC-Kenya; Dr. Bunnell is the associate director for science at CDCUganda; and Dr. Mermin is the director of CDC-Uganda. The other participants in the Kenya-Uganda HIV Testing Group were Elizabeth Marum, Ph.D., Barbara Marston, M.D., Dorothy Mbori-Ngacha, M.B., Ch.B., M.Med., and Laurence Marum, M.D., M.P.H., in Kenya, and Donna Kabatesi, M.B., Ch.B., M.P.H., in Uganda.
\end{abstract}

1. UNAIDS/WHO policy statement on HIV testing. Geneva: Joint United Nations Programme on HIV/AIDS, June 2004.

2. The right to know: new approaches to HIV testing and counseling. Geneva: World Health Organization, 2003.

3. Central Bureau of Statistics (CBS) [Kenya], Ministry of Health (MOH) [Kenya], ORC Macro. Kenya demographic and health survey 2003. Calverton, Md.: ORC Macro, July 2004:227-8.

4. Weller S, Davis K. Condom effectiveness in reducing heterosexual HIV transmission. Cochrane Database Syst Rev 2001;3: CD003255.

5. Downing RG, Otten RA, Marum E, et al. Optimizing the delivery of HIV counseling and testing services: the Uganda experience using rapid HIV antibody test algorithms. J Acquir Immune Defic Syndr Hum Retrovirol 1998;18:384-8. 